\title{
Mamillothalamic Tract Transection Blocks Anterior Thalamic Training-induced Neuronal Plasticity and Impairs Discriminative Avoidance Behavior in Rabbits
}

\author{
Michael Gabriel, Cyndi Cuppernell, Joel I. Shenker, Yasuo Kubota, Victor Henzi, ${ }^{a}$ and Douglas Swanson ${ }^{b}$ \\ Department of Psychology and Beckman Institute, University of Illinois at Urbana-Champaign, Urbana, Illinois 61801
}

\begin{abstract}
Rabbits with bilateral transecting lesions of the mamillothalamic tract, control (tract-sparing and sham) lesions, or no lesions, and chronic, fixed-position anterior ventral (AV) and medial dorsal (MD) thalamic and posterodorsal subicular complex unit recording electrodes were trained to step in an activity wheel in response to a $0.5 \mathrm{sec}$ tone $(\mathrm{CS}+)$ in order to avoid a brief foot shock. The rabbits also learned to ignore a different tone (CS-) not predictive of shock. Behavioral acquisition was significantly retarded in rabbits with mamillothalamic tract transection compared to controls. When trained, transected rabbits failed to avoid the shock more often than controls. Mamillothalamic tract transection abolished and control lesions attenuated AV thalamic discriminative training-induced activity (i.e., development with training of greater discharges in response to the CS+ than to the CS-). Transection and control lesions attenuated AV thalamic excitatory training-induced activity (greater elicited activity during training than during unpaired tone-shock presentations before training) as well as AV thalamic "spontaneous" baseline unit activity. CS-elicited discharge magnitude was reduced by control lesions and it was further reduced by tract transecting lesions. Significant lesion-related changes were not found in the subicular or MD thalamic neuronal records. Mamillothalamic tract afferent information flow is thus essential for AV thalamic discriminative training-induced activity, excitatory training-induced activity, tone-elicited discharges and maintenance of conditioned avoidance responses. The effects of the control lesions suggested that afferents which course in parallel with and near the mamillothalamic tract may contribute to $\mathrm{AV}$ thalamic spontaneous activity and excitatory training-induced activity.

[Key words: training-induced neuronal activity, dynamic neuronal plasticity, instrumental conditioning, avoidance learning, limbic system, memory, functional brain circuitry, in vivo unit recording, significance coding, activity-dependent facilitation, muscarinic $M_{2}$ receptors]
\end{abstract}

Analyses of the neural mediation of learning and memory are advancing in relation to neurobiology of activity-related synaptic strength adjustments (Madison et al., 1991; Massicotte and

Received Dec. 31, 1993; revised July, 1994; accepted Aug. 12, 1994.

This research was supported by NIH Grant NS26736, NIMH Grant MH37915, and NSF Grants IBN 88-21219 and BIR 9I-16763.

Correspondence should be addressed to M. Gabriel, Beckman Institute, University of Illinois, 405 North Mathews Avenue, Urbana, IL 61801.

"Present address: Department of Physiology and Cell Biophysics, Columbia University, BB 1106, 630 West 168th Street, New York, NY 10032

'Present address: Department of Neuroscience, University of Florida, Gaincsville FL 32610 .

Copyright (c) 1995 Society for Neuroscience $0270-6474 / 95 / 151437-09 \$ 05.00 / 0$
Baudry, 1991; Bramham, 1992; Johnston et al., 1992; Colley and Routtenberg, 1993), analyses of circuitry and fundamental mechanisms of plasticity underlying invertebrate learning (e.g., Hawkins et al., 1993) and the elucidation of the circuitry and neuronal information processing in a variety of mammalian learning paradigms (Gabriel and Moore, 1990).

The present study continues an ongoing analysis in the third category, of discriminative instrumental avoidance learning, wherein rabbits learn to step (in a large activity wheel) in response to a warning tone ( $\mathrm{CS}+$ ) in order to avoid a foot-shock, and they learn to ignore a different tone (CS-) which does not predict shock. Lesion studies have implicated circuitry of the limbic thalamus [the anterior and medial dorsal (MD) nuclei], the cingulate cortical projection fields of these nuclei, and the hippocampal formation in this learning (Gabriel et al., 1989, 1991), and massive changes of unit activity in response to the $\mathrm{CS}+$ and CS - develop in these areas during avoidance training. This training-induced activity is both excitatory (greater during training with paired CS-US presentations than before training with unpaired tone and US presentations) and discriminative (greater in response to the CS+ than to the $\mathrm{CS}-$ in trained rabbits).

Additional work has indicated that thalamic training-induced activity does not require input from cortex, whereas cingulate cortical training-induced activity does require thalamic input. Experimental damage to subicular and cingulate cortical neurons that project to limbic thalamus did not attenuate but rather enhanced the limbic thalamic training-induced activity (Gabriel et al., 1986, 1987, 1991). Lesions of the limbic thalamic nuclei abolished training-induced activity and virtually all tone-elicited activity in cingulate cortex (Gabriel et al., 1989). Therefore, CSelicited neuronal activation relevant to the performance of the learned behavior flows from thalamus to cortex, not from cortex to thalamus. These results set the stage for a search for the subcortical afferents which are critical for the development of the thalamic training-induced activity.

Microinfusion of 6-hydroxydopamine depleted anterior thalamic nor-epinephrine (NE) to within $4 \%$ of control values but did not reduce the thalamic training-induced activity. Indeed, training-induced activity was enhanced by this manipulation (Sparenborg and Gabriel, 1992). Thus, projections of NE-containing brainstem neurons to the anterior thalamus do not appear to be essential for the thalamic training-induced activity (although the depletion of NE did interfere with anterior thalamic neuronal processing of unexpected stimuli).

The ACh-containing fibers which project to the anterior nuclei from the lateral dorsal tegmental nucleus (e.g., Satoh and Fibiger, 1986) may contribute specifically to anterior ventral (AV) 
thalamic excitatory training-induced activity, as the elicited firing of AV thalamic neurons in trained rabbits was greatly attenuated following systemic administration of the muscarinic receptor antagonist scopolanine hydrobromide. Intriguingly, AV thalamic discriminative training-induced activity was not affected by the scopolamine injections (Henzi et al., 1990).

The present study addressed the relevance of the massive mamillothalamic afferent projection system for learning and AV and MD thalamic training-induced activity. Multiunit activity was recorded in these nuclei during avoidance training in controls and in rabbits given electrolytic lesions which transected the mamillothalamic tract. The lesions were made prior to training, during surgery for implantation of recording electrodes.

\section{Materials and Methods}

Subjects, electrodes, surgery, and recording procedures. The subjects were 49 male New Zealand White rabbits weighing $1.5-2.0 \mathrm{~kg}$ on delivery to the laboratory and maintained on ad libitum water and a regimen of rabbit chow ( 1 cup daily) restricted to prevent obesity. After a minimum period of $48 \mathrm{hr}$ for adaptation to living cages, each rabbit underwent surgery for implantation of unit recording electrodes. Surgical anesthesia was induced by either injection of chlorpromazine (12.5 $\mathrm{mg})$ in solution followed by sodium pentobarbital $(25.0 \mathrm{mg})$ in solution through the marginal vein of the pinna or subcutaneous injection $(1 \mathrm{ml} /$ $\mathrm{kg}$ of body weight) of a solution containing $60 \mathrm{mg} / \mathrm{ml}$ of ketamine $\mathrm{HCl}$ and $8 \mathrm{mg} / \mathrm{ml}$ of xylazine followed by hourly injections of $1 \mathrm{ml}$ of the solution. During surgery, bilateral electrolytic lesions of the mamillothalamic tract were attempted in 23 rabbits. The lesions were made by passing a 1.0-1.5 mA cathodal D.C. current for $15 \mathrm{sec}$ through lesioning electrodes made from stainless-steel insect pins coated with an insulating material (Epoxylite). The insulator was removed from the tips to form $0.7-1.0 \mathrm{~mm}$ surfaces for passage of current.

The fibers of mamillary neurons give rise to two major tracts, the mamillothalamic and the mamillotegmental tracts. The fibers which form the mamillotegmental tract project rostralward in the caudoventral hypothalamus, before they exit from the main tract and project caudally (Shen, 1983). The stereotaxic coordinates used for the mamillothalamic tract lesions ( $\mathrm{AP}=+2.5, \mathrm{~L}= \pm 1.5, \mathrm{~V}=10.3$; Girgis and Shih-Chang, 1981) defined a site rostral to the site at which the mamillotegmental tract separates from the mamillothalamic tract. In four of the rabbits, the lesions were made at the same AP and $\mathrm{L}$ coordinates, but two vertical coordinates were used $(10.0$ and $11.0,10.5$ and 11.5 , or 10.3 and 10.8).

Four or six stationary multiunit recording electrodes were implanted in each rabbit through burr holes (diameter $=0.5 \mathrm{~mm}$ ) drilled through the skull over the target sites. Various target-site distributions in each rabbit were employed to attain an equal number of recordings for the experiment as a whole, in the following sites: the AV thalamic nucleus $(\mathrm{AP}=+2.0, \mathrm{~L}= \pm 2.3$ and $\mathrm{V}=7.5)$, the $\mathrm{MD}$ thalamic nucleus (AP $=+4.6, \mathrm{~L}= \pm 1.5, \mathrm{~V}=8.25$ ), the dorsoposterior subicular complex $(\mathrm{AP}=+8.5, \mathrm{~L}= \pm 6.75, \mathrm{~V}=4.5$ ), the medial mamillary nuclei (AP $=4.5$ or $5.5 ; \mathrm{L}=0.5, \mathrm{~V}=14.0$ or 14.5 ), and areas $\mathrm{CA} 1, \mathrm{CA} 3$, and the dentate gyrus of Ammon's horn. The recording electrodes were fabricated as were the electrodes for making lesions, but with smaller exposed tips (10-50 $\mu \mathrm{m} ; 1 \mathrm{kHz}$ impedance: $500 \mathrm{k} \Omega$ to $2 \mathrm{M} \Omega$ ). Miniature Teflon cylinders (length $=2.5 \mathrm{~mm}$; diameter $=1.5 \mathrm{~mm}$ ) impaled on stainless stecl insect pins were positioned over cach burr hole and affixed to the skull using dental acrylic. The pins were removed from the cylinders after hardening of the acrylic. Recording electrodes were then slowly advanced to the recording sites by press fitting them through the holes in the Teflon cylinders. Unit activity was monitored during electrode advancement to enhance placement accuracy.

Behavioral training. Following a minimum period of one week for recovery from surgery, the rabbits received training in an activity wheel designed for the administration of aversive conditioning (Brogden and Culler, 1936). The wheel was contained in a shielding chamber in a room adjacent to that housing the equipment used for data collection. A video camera and monitor permitted viewing of the rabbit during conditioning. An exhaust fan and a speaker in the chamber produced a masking noise of $70 \mathrm{~dB}$ re $20 \mathrm{~N} / \mathrm{m}^{2}$, throughout training. The conditional stimuli were pure tones ( 1 or $8 \mathrm{kHz}$, free field, $85 \mathrm{~dB}$ re $20 \mathrm{~N} / \mathrm{m}^{2}$, rise time $=3 \mathrm{msec}$ ) played through a speaker attached to the chamber ceiling directly above the wheel. Onset of the positive conditional stimulus (CS+) was followed after $5 \mathrm{sec}$ by the shock unconditional stimulus (a constant current 1.5-2.5 mA foot shock delivered through the grid floor of the wheel). The shock was terminated by behavioral responses, defined as wheel rotations exceeding $2^{\circ}$. Responses after $\mathrm{CS}+$ onset but prior to shock onset prevented shock administration. The duration of the CS+ was 0.5 sec. The maximum duration of the shock was $1 \mathrm{sec}$. The $\mathrm{CS}-$ was a $0.5 \mathrm{sec} 1 \mathrm{kHz}$ or $8 \mathrm{kHz}$ tone, the tone not chosen as CS+. The CS- was never followed by the shock. The interval from the end of a trial (defined as the end of the $5 \mathrm{sec}$ period following CS onset, or of wheel rotation when locomotion occurred) to CS onset for the next trial was $8,13,18$, or $23 \mathrm{sec}$. These values occurred in an irregular sequence. Responses during the intertrial interval reset the interval.

The rabbits received daily 60 trials with the CS + and 60 trials with the CS -, in an irregular sequence, until a criterion of performance was reached. The criterion required that the percentage of trials with behavioral responses to the CS+ (i.e., avoidance responses) exceed the percentage of trials with responses to the CS- by 60 or more, in two consecutive sessions. For example, a rabbit would meet this criterion by performing conditioned responses on $80 \%$ of the $\mathrm{CS}+$ trials and on $20 \%$ of the CS - trials, or by performing conditioned responses on $90 \%$ of the CS + trials and on $30 \%$ of the CS - trials. This criterion yields an asymptote of performance, that is, performance levels achieved at criterion do not change significantly during postcriterial training. Training was terminated if criterion was not attained after 15 sessions.

Prior to training, each rabbit received two preliminary training sessions, in which CS + and $\mathrm{CS}-$ were presented, each 60 times. The intervals between the tones were timed as during training. The tones only were presented in the first of these sessions and the tones with explicitly unpaired shock presentations were given in the second session (Rescorla, 1967), that is, shock was not presented during the tone, within $3 \mathrm{sec}$ prior to the tone, or within $7 \mathrm{sec}$ after the tone. The incidence and distribution over trials of the shock during this session were identical to the average values of these parameters obtained during the initial session of training for a sample of 100 rabbits. Because the CS did not predict the shock in this session, avoidance learning did not occur. This session thus yielded baseline data for detecting associative neural and behavioral changes brought about by pairing the $\mathrm{CS}+$ with the shock during training.

Collection of neuronal data. The neuronal records were fed into fieldeffect transistors (FETs) attached to the connector which mated with the cranial socket, about $2.5 \mathrm{~cm}$ from the brain recording sites. The FET outputs were led from the rabbit through the axle of the rotating wheel and the wall of the conditioning chamber via a shielded cable. Each recording channel was split, one limb entering single-ended preamplifiers with bandwidth appropriate for unit recording ( $1 / 2$ amplitude cutoffs at 500 and $8000 \mathrm{~Hz}$ ) the other limb entering preamplifiers for electroencephalographic recording of field potentials ( $1 / 2$ amplitude cutoff at 0.2 and $60 \mathrm{~Hz}$ ). The unit activity was subjected to a second stage of active bandpass filtering (1/2 amplitude cutoff at 600 and $8000 \mathrm{~Hz}$, rolloff $=18 \mathrm{~dB} /$ octave) to remove all slow field potential frequencies. The filter outputs entered Schmitt triggers, which produced an $80 \mu \mathrm{sec}$ square wave output pulse when the input voltage exceeded a preset threshold. The thresholds were adjusted under computer control to yield a mean output pulse rate within limits of 95-165 per second. With this criterion, typically, the largest three or four spikes were sampled. In addition, the bandpass filter outputs were half-wave rectified and integrated (see Buchwald et al., 1973). The time constants for the rise and fall of the integrators were 15 and $75 \mathrm{msec}$, respectively. The Schmitttrigger data indicated the discharge frequency of the largest spikes on each record. The integrated unit activity measured energy fluctuations of the entire record, including activity below the triggering thresholds.

The Schmitt trigger events were counted and the integrator outputs and field potentials digitized in $10010 \mathrm{msec}$ intervals, $0.3 \mathrm{sec}$ before and $0.7 \mathrm{sec}$ after CS-onset. A digital value was stored for each measure and electrode, every $10 \mathrm{msec}$ during the sampling interval. Only the spike frequency and integrated unit activity data are presented in this report.

Due to the smoothing effect of the time constants, the integrated unit activity yields somewhat less accurate CS-elicited neuronal discharge profiles than are yielded by the spike frequency data. However, the integrated unit activity measure is more sensitive than the spike frequency measure and on occasion reveals significant rclationships not seen or seen only as nonsignificant trends in the spike frequency data. 


\section{MTT Lesions}
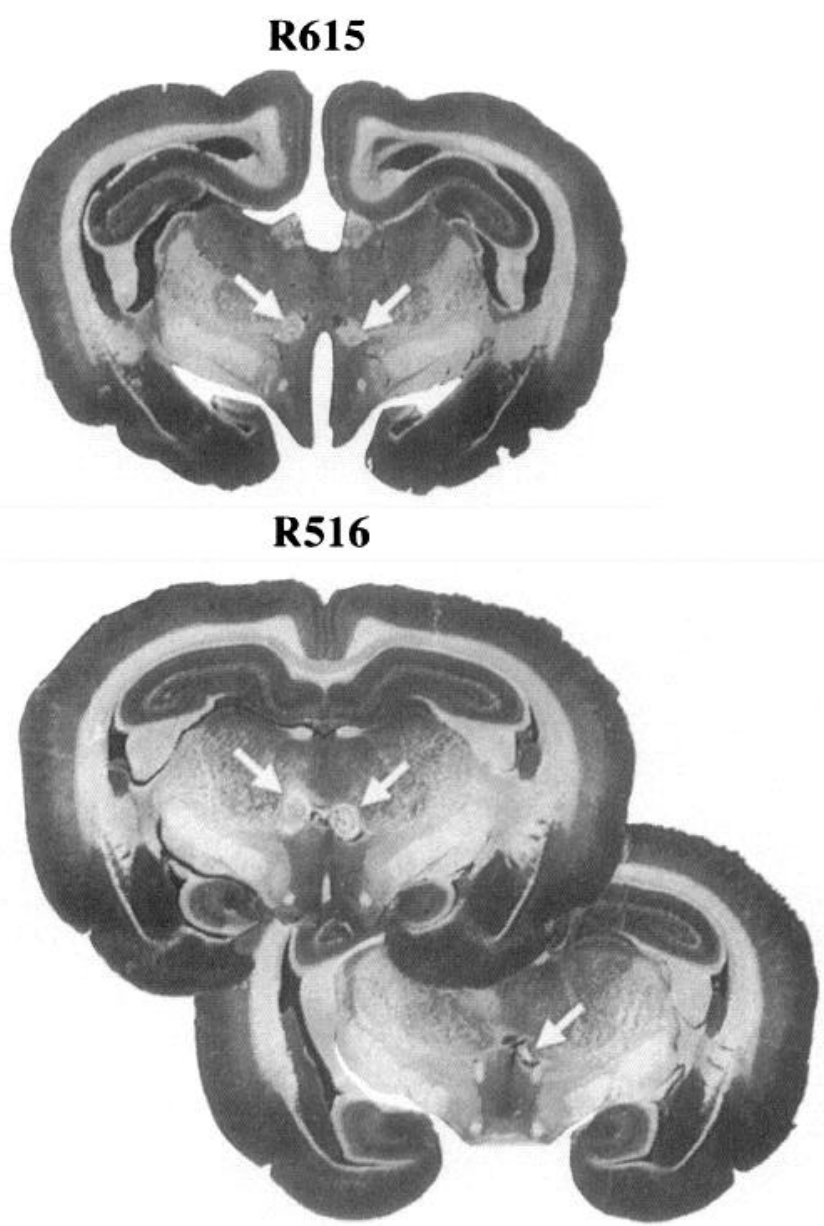

MTT-Sparing Lesions
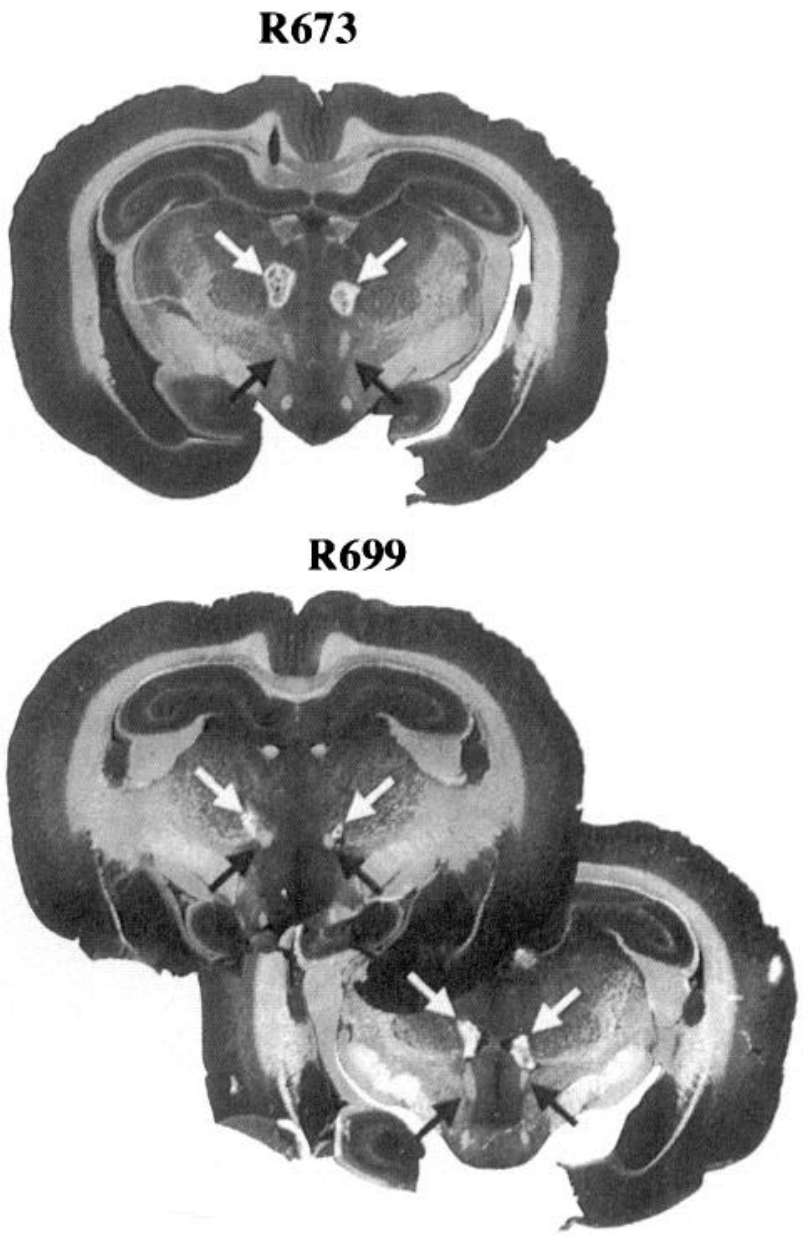

Figure 1. Coronal sections with typical bilateral electrolytic lesions in the mamillothalamic tract (MMT, left) and lesions which spared the mamillothalamic tract (right). Lesions are indicated by white arrows, and spared mamillothalamic tract by black arrows. Sections from a rabbit with small lesions (top) and one with large lesions (bottom) are shown for each lesion type.

Here, the spike frequency data are presented for all analyses in which the same significant relationships were revealed by both measures. The statistically significant effects obtained in analyses of the integrated unit activity are given for instances in which the effects were not significant but present as nonsignificant trends in the analysis of the spike frequency data.

Several computer- and experimenter-controlled methods were used for exclusion of data samples containing movement artifacts (see Gabriel et al., 1983)

Data analysis. Varied numbers of training sessions were required for criterion attainment. The analysis of the neuronal data focused on behaviorally defined stages of training common to all rabbits. Each stage was represented by the data of a single training session. The stages were (1) preliminary training with explicitly unpaired presentations of the CSs and foot shock, (2) the first session of avoidance training, (3) the session in which the first significant behavioral discrimination was performed, (4) the session in which the acquisition criterion was attained. The session of the first significant behavioral discrimination was the avoidance training session in which the percentage of locomotions performed in response to the CS + first exceeded by $25 \%$ or more the percentage of locomotions performed in response to the CS-.

In order to obtain a measure of the unit activity elicited by the CS+ and $\mathrm{CS}-$, the difference between the mean sampled activity values in the 30 pre-CS baseline $10 \mathrm{msec}$ intervals and the values in each of the first 40 intervals after CS onset was calculated. This procedure differs from our standard $z$-score calculation, which involves an additional step of dividing the differences by the standard deviation of the baseline values. However, use of the standard deviation was precluded for the present data as the standard deviations were found to be systematically affected by the lesions, as reported below.

The neuronal and behavioral data were submitted to factorial, repeated measure analysis of variance (ANOVA) using the $\mathrm{P} 2 \mathrm{~V}$ program (BMDP Statistical Software, Inc.). The alpha level for all testing was set at 0.05 . The analyses had factors of groups [three levels: mamillothalamic tract transected, control (sham and tract-sparing) lesions, and no lesion as described below], training stages (four levels as described above), stimulus ( 2 levels: $\mathrm{CS}+/ \mathrm{CS}-$ ), and, for the neuronal data, post$\mathrm{CS}$ interval (40 levels). Factors yielding significant overall $F$ ratios in the analysis of variance were further analyzed using simple effect tests following procedures outlined by Winer (1962, Chap 7). Correction of the $F$ test due to violations of the sphericity assumption of these analyses was performed as needed following the procedure of Huynh and Feldt (1976). The uncorrected $F$ ratios reported in the text represent only those effects which were significant after correction.

Histology, lesion assessment, and experimental groups. After the completion of training, a lethal dose of pentobarbital sodium was followed by transcardiac perfusion with normal saline and $10 \%$ formalin. The brains were removed, frozen and sectioned at $40 \mu \mathrm{m}$, and the sections were photographed while still wet (Fox and Eichman, 1959). The dried sections were treated with a metachromatic nissl and myelin stain using formol-thionin (Donovick, 1974).

Nine of the rabbits in which lesions were attempted had complete 


$\begin{aligned} & \text { Table 1. The number and location of neuronal records in the } \\
& \text { three experimental groups }\end{aligned}$
\begin{tabular}{lccc} 
Recording site & Lesion & Control lesion & No lesion \\
\hline AV Nucleus & 14 & 12 & 18 \\
MD nucleus & 5 & 5 & 5 \\
Posterodorsal subiculum & 4 & 3 & - \\
Presubiculum & 3 & 5 & - \\
\end{tabular}

bilateral transection of the mamillothalamic tract (Fig. 1), four had partial bilateral mamillothalamic tract damage and three had unilateral mamillothalamic tract transection. These rabbits were included in a "lesion group" $(N=16)$ for assessment of the behavioral effects of the lesions. Because partial and unilateral mamillothalamic tract lesions which affected behavior may have failed to alter particular recordings of thalamic neuronal activity, only the neuronal records of rabbits with complete bilateral transection of the mamillothalamic tract $(N=9)$ were used for analyses of the neuronal data.

The lesions missed the mamillothalamic tract, but did damage areas surrounding the mamillothalamic tract in seven rabbits. The data of these rabbits were pooled with data of five rabbits given sham lesions (electrode insertion without current passage) to form a "control lesion" group $(N=12)$. Preliminary analyses comparing the behavioral and neuronal data of the rabbits with sham and tract-sparing lesions did not yield significant differences, or any trends suggesting that differences would be found with larger samples. In order to assess the effects of the control lesions, neuronal records of rabbits that served as controls in other studies, with identical parameters of behavioral training and AV thalamic recording electrodes, were assigned to a group with no lesions $(N=16)$. The data of the no-lesion rabbits were obtained concurrently, over a period of several years, with the data of the rabbits given lesions. A separate no-lesion group $(N=5)$ with MD thalamic recording electrodes was used in the analyses of the MD thalamic data. The photographs of the wet sections containing electrode tracks indicated $44,15,7$, and 8 recording probes in the AV nucleus, MD nucleus, posterodorsal subiculum and presubiculum, respectively (see Table 1). No significant effects of the lesions were found on MD thalamic or subicular neuronal activity, and thus the records of these areas are not considered further in this report. Insufficient data were obtained from the mamillary and Ammon's horn recording sites. This report thus focuses exclusively on the effects of the lesions on the neuronal activity of the AV thalamic nucleus.

\section{Results}

Mamillothalamic lesions and avoidance behavior

Discriminative avoidance learning was significantly retarded in rabbits with mamillothalamic tract lesions. These rabbits required an average of 8.44 training sessions to meet the criterion, whereas the rabbits with control lesions and no lesions met the criterion in 6.17 and 4.94 sessions, respectively. The analysis yielded a significant main effect $[F(2,41)=3.26, P<0.048]$ of the grouping factor. Individual comparisons indicated that the mean number of sessions taken by rabbits with lesions to reach the criterion exceeded significantly the mean for the rabbits with no lesions $(P<0.05)$. The rabbits with control lesions did not differ significantly from either the rabbits with no lesions or the rabbits with lesions.

The incidence of successful avoidance responses during criterion attainment in rabbits with lesions was significantly lower than that exhibited by rabbits with control lesions and no lesions (Fig. 2).

An analysis was computed on the percentage of trials (CS presentations) in which locomotory responses were performed, in each of four stages of behavioral acquisition (pretraining, the first conditioning session, the session of the first significant discrimination and the session in which criterion was attained).

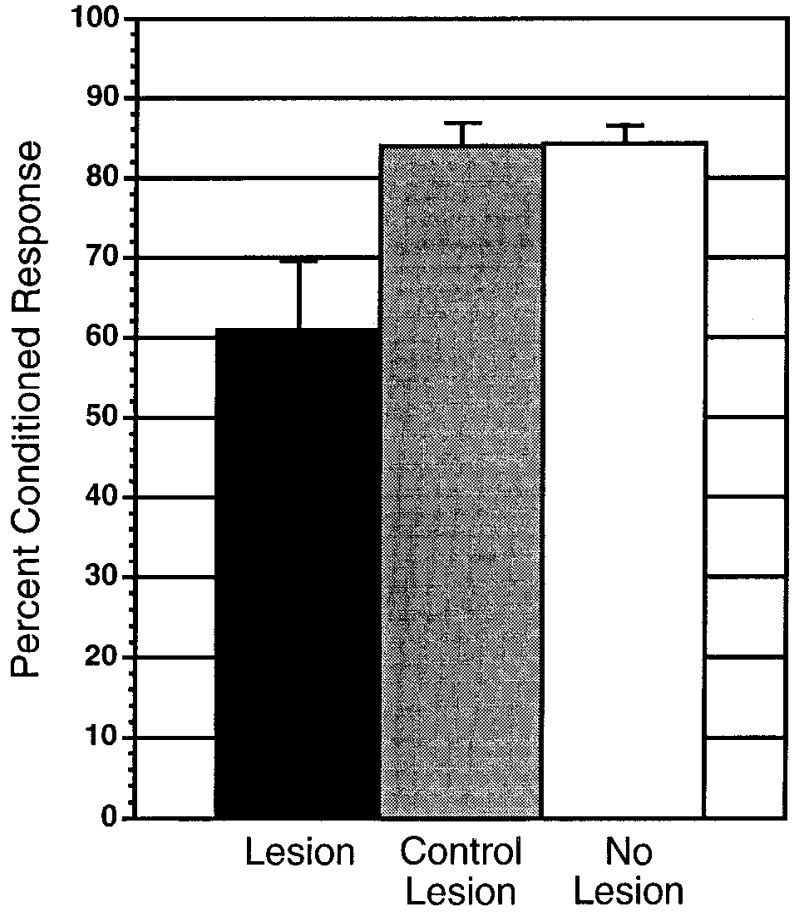

Figure 2. The average percentage of avoidance responses performed by rabbits with mamillothalamic tract lesions, rabbits with control lesions and no-lesion controls in response to the CS + presentations during the session in which the acquisition criterion (described in Materials and Methods) was attained.

Simple effect tests following a significant interaction of the factors of lesion, acquisition stage and stimulus [CS +/CS - ; $F(6,123)=2.98, P<0.01]$ showed that the performance levels of the three groups did not differ during preliminary training, the first conditioning session or during the session in which the first significant behavioral discrimination occurred. However, the rabhits with control and no lesions exhibited significantly greater average percentages $(83.9 \%$ and $84.2 \%$, respectively) of trials with avoidance responses than did the rabbits with lesions $(60.9 \%)$ during the session in which the criterion was attained $(P<0.01)$. No significant differences were found between the groups in the incidence of responses to the CS- .

In summary, the mamillothalamic tract lesions retarded but did not prevent behavioral acquisition. In addition, the lesions reduced the incidence of successful avoidance responses by trained rabbits.

Additional analyses did not indicate significant effects of the lesions on the latencies or durations of conditioned and unconditioned (shock-elicited) responses or on the numbers of intertrial responses performed during acquisition.

The aforementioned effects of the lesions were very similar when the analysis was restricted to the reduced subset of rabbits $(N=9)$ with bilaterally transected mamillothalamic tracts. Upon repeating these analyses using for the lesion condition the behavioral records of only the bilaterally transected rabbits, a significant loss of performance efficiency in trained rabbits $(P<$ $0.01)$ was again found. In addition, there was a trend indicating retardation of acquisition in this group: the number of sessions needed to attain criterion by the transected rabbits was $8.00 \mathrm{com}$ pared with 6.17 and 4.94 in the control lesion and no-lesion groups. Nevertheless, these values did not yield a significant $F$ 
due to the slight drop of the mean from 8.44 to 8.00 in the lesion condition, and the reduction of the sample size.

\section{Mamillothalamic tract lesions and neuronal activity}

Baseline activity. Analyses were performed on the integrated unit activity recorded during the $3010 \mathrm{msec}$ intervals comprising the baseline period prior to each CS presentation. The mean AV thalamic baseline integrated activity was $32.1(3.85 \mu \mathrm{V})$, $41.6(5.08 \mu \mathrm{V})$, and $66.5(8.12 \mu \mathrm{V})$ for the lesion, control-lesion and no-lesion groups, respectively, yielding a significant main effect in the analysis of variance $[F(2,41)-12.01, P<0.0002]$. Individual comparisons demonstrated that the baseline means yielded by transected rabbits and by rabbits with control lesions were significantly reduced relative to the means of the rabbits with no lesions $(P<0.01)$. The transected rabbits did not differ significantly from those with control lesions.

The lesions also reduced the variability of the AV thalamic baseline activity, as indicated by an analysis of the SD of the activity in the $3010 \mathrm{msec}$ pre-CS intervals $[F(2,46)=11.22$, $P<0.0002]$. The average values of the SDs in rabbits with lesions, control lesions and no lesions were $0.30,0.33$, and 0.71 , respectively. Again, the means of the transected rabbits and rabbits with control lesions did not differ significantly, but were significantly reduced relative to the means of rabbits with no lesions $(P<0.01)$. These results indicated that mamillothalamic tract afferents and/or nonmamillothalamic tract afferents in the vicinity of the mamillothalamic tract, which were disrupted by the mamillothalamic tract and control lesions, are essential for maintenance of the level of ongoing baseline activity of $\mathrm{AV}$ thalamic neuronal ensembles. Also, as indicated in Materials and Methods, the lesion-related alterations of the baseline variation called for the use of simple subtraction, rather than the standard $\mathrm{z}$-score calculation, to normalize neuronal activity which followed CS onset.

Sensory discharges elicited by the conditional stimuli. As in past studies (Gabriel, 1993) the CS+ and CS- elicited triphasic average neuronal discharges in the AV thalamic nucleus, consisting of (1) excitation, reaching maximum $20-30 \mathrm{msec}$ after CS onset, (2) an inhibitory pause from 40 to $70 \mathrm{msec}$, and (3) excitation from $90 \mathrm{msec}$ until the end of the $400 \mathrm{msec}$ sampling epoch. The triphasic profile is shown in the second and third row of panels in Figure 3.

The triphasic AV thalamic histogram profiles were attenuated in rabbits with transected mamillothalamic tracts and control lesions, relative to the profiles of rabbits with no lesions (compare the panels in the first row to those in the second and third rows of Fig. 3). In addition, the histogram profiles of rabbits with lesions were significantly attenuated relative to the profiles of rabbits with control lesions (compare the panels in the first row to those of the second row in Fig. 3). Moreover, the inhibitory pause after CS onset present in the rabbits with control and no lesions was abolished in transected rabbits (compare the panels in the first row to those in the second and third rows of Fig. 3). These changes were indicated by significant interactions of the Lesion and post-CS Interval factors $[F(78,1521)=3.29, P<$ 0.0001 , spike frequency; $F(78,1521)=9.06, P<0.0001$, integrated activity]. These interactions did not include the factors of training stage or stimulus, indicating that the lesion-related changes were not specific to particular training stages or eliciting stimuli.

Simple effect tests carried out for the spike frequency data demonstrated that the average AV thalamic neuronal discharges of transected rabbits were significantly reduced, compared to the discharges of rabbits with no lesions, in virtually all $10 \mathrm{msec}$ intervals from 20 to $400 \mathrm{msec}$ after CS onset. The only exception occurred for intervals 5 through 11 , from 40 to $110 \mathrm{msec}$ after CS onset, wherein the average discharges of transected rabbits exceeded significantly those of the rabbits with no lesions, this due to the abolition of the inhibitory pause in the histogram profiles of transected rabbits. Also, the average discharges of transected rabbits were significantly reduced, relative to those of rabbits with control lesions. For this comparison, the differences were confined to intervals from 220 to $400 \mathrm{msec}$ after CS onset. However, again, the average activity from 30 to 90 msec after CS onset present in transected rabbits exceeded the activity in rabbits with control lesions during these intervals, due to the absence of the inhibitory pause in the transected rabbits. Finally, the activity in the rabbits with control lesions was significantly reduced relative to the activity in the rabbits with no lesions at all intervals from 160 to $400 \mathrm{msec}$ after CS onset. There were, however, two intervals (from 20 to $40 \mathrm{msec}$ after CS onset, representing the initial bricf-latency excitatory component of the triphasic discharge profile) at which the activity of rabbits with control lesions exceeded significantly the activity of rabbits with no lesions. The probability levels for these comparisons varied from the criterion level of $P<0.05$ to $P<$ 0.0001 .

\section{Training-induced neuronal activity}

Excitatory activity. Excitatory training-induced activity refers to enhancement of the neuronal activity elicited by the conditional stimuli during training relative to the elicited activity during preliminary training with unpaired CS and shock presentations. An effect of the lesions on AV thalamic excitatory training-induced activity was indicated by significant interactions of the factors of lesion, stimulus and training stage $[F(6,117)=2.68, P<$ 0.02 , spike frequency; $F(6,117)=3.00, P<0.0093$, integrated activity].

Individual comparisons carried out for the spike frequency data indicated that the expected AV thalamic excitatory training-induced activity did occur in rabbits with no lesions, in the form of a significantly greater average discharge in response to the CS+ during the criterial session of acquisition (Fig. 3, third row, second panel, solid bars) compared to the average discharge during the preliminary training session (Fig. 3, third row, first panel, solid bars; $P<0.01$ ). However, excitatory training-induced activity did not occur in any training session in transected rabbits or in rabbits with control lesions. Indeed, the average excitatory discharges elicited by the CS + and by the CS - in the transected rabbits were significantly attenuated during the criterial stage of acquisition (Fig. 3, top row, second panel), relative to the preliminary training session $(P<0.05$; Fig. 3, top row, first panel).

Similarly, the average discharges elicited by the CS - in rabbits with control lesions were significantly attenuated $(P<$ 0.05 ) in the criterial session (Fig. 3, second row, second panel, open bars), relative to preliminary training (Fig. 3, second row, first panel, open bars). The discharges elicited by the CS + of rabbits with control lesions did not change during training (Hig. 3 , second row, solid bars).

Discriminative activity. Discriminative training-induccd activity refers to the development of significantly different neuronal discharge profiles in response to the CS + than to the CS - during training. None of the groups exhibited significant 


\section{Spike Frequency}

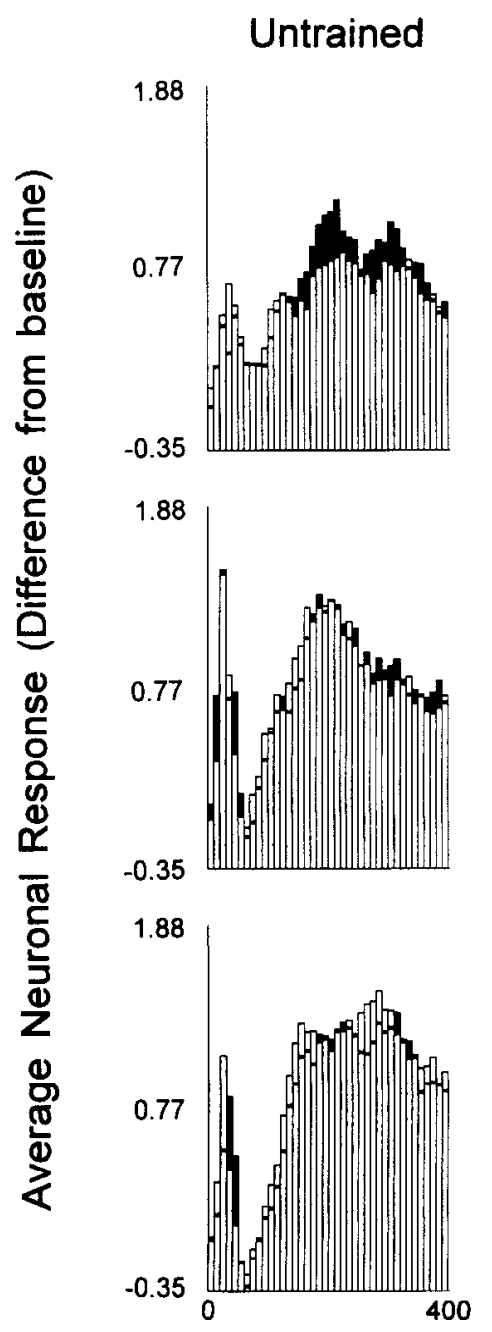

Trained
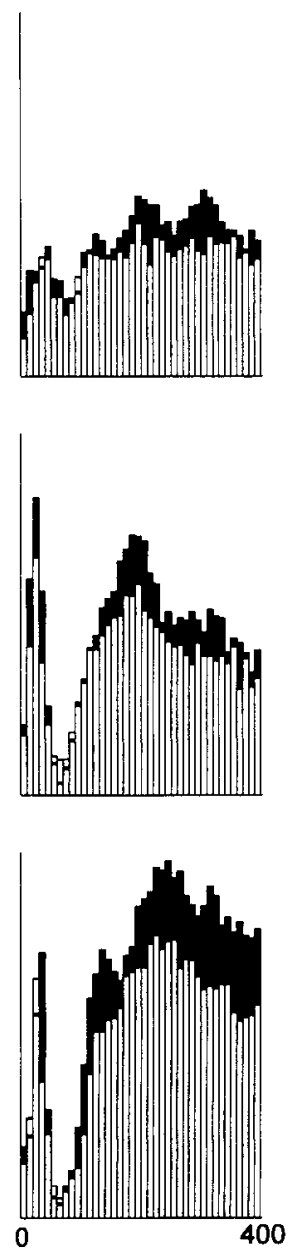

Integrated Unit Activity

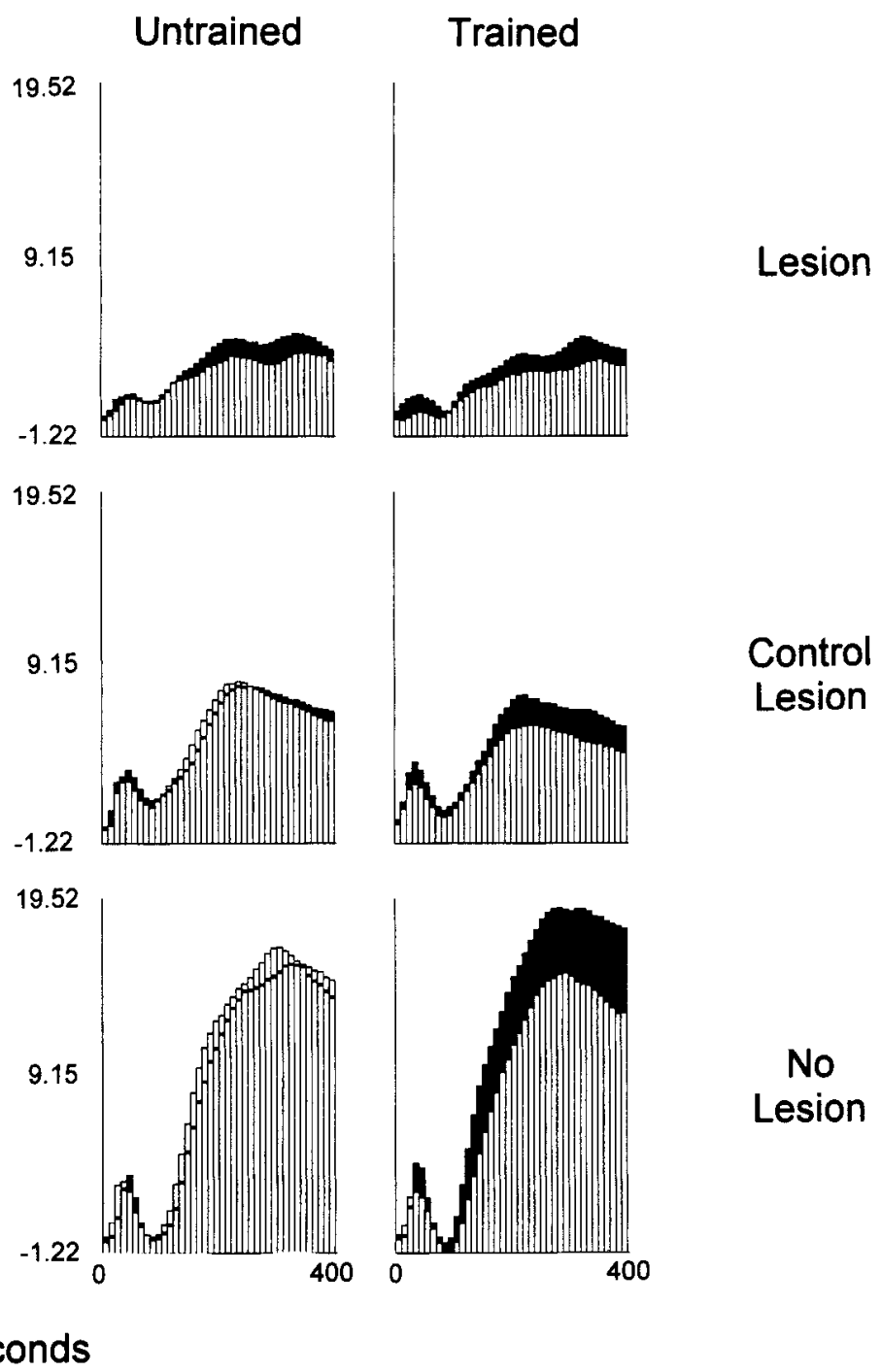

Figure 3. Average multiunit spike frequency (left panels) and integrated activity (right panels) profiles in the AV nucleus in response to CS + (solid bars) and $\mathrm{CS}-$ (open bars), in rabbits with mamillothalamic tract lesions, rabbits with control lesions and no-lesion controls. Each panel shows the unit activity during the first 40 consecutive $10 \mathrm{msec}$ intervals following CS onset. The plotted values are difference scores: the result of subtracting the discharge magnitude in each $10 \mathrm{msec}$ interval after CS onset from the mean score of the 30 pre-CS intervals. The records were obtained during the preliminary training session (Untrained) and the session in which the acquisition criterion was attained (Trained), as described in Materials and Methods.

discriminative training-induced activity during preliminary training (Fig. 3, first and third columns). As in past studies, significantly greater excitatory discharges in response to the $\mathrm{CS}+$ than to the $\mathrm{CS}$ - occurred during the criterial session of acquisition in the rabbits with control lcsions $(P<0.05)$ and no lesions $(P<0.01$; Fig. 3 , second and third rows, second panel). However, this effect did not occur in rabbits with bilateral transection of the mamillothalamic tract (Fig. 3, first row, second panel). Also, as is characteristic of AV thalamic neuronal activity, significant discriminative training-induced activity did not occur during the first session of conditioning or during the session of the first significant behavioral discrimination. This finding replicates previous findings of exclusively "late-developing" discriminative activity in the AV nucleus
(Gabriel et al., 1977, 1980; Foster et al., 1980) of intact rabbits.

The occurrence of discriminative training-induced activity during criterial performance in the no-lesion and control-lesion rabbits, and its nonoccurrence in transected rabbits, were corroborated by separate analyses of the data of each group. The interactions of the factors of training stage and stimulus, which assessed discriminative training-induced activity, were significant for the no-lesion and control-lesion groups $[F(3,51)=8.79$, $P<0.0002$, no lesion, spike frequency; $F(3,51)=6.71, P<$ 0.0008 , no lesion, integrated activity; $F(3,33)=5.19, P<$ 0.0049 , control lesion, spike frequency; $F(3,33)=5.92, P<$ 0.0025 , control lesion, integrated activity] but not for the transected rabbits $[F(3,39)=0.51, P<0.69$, spike frequency; $F(3,39)=1.42, P<0.26$, integrated activity]. 


\section{Discussion}

Neuronal populations of the anterior ventral (AV) thalamic nucleus in rabbits exhibit training-induced excitation, and discrimination between $\mathrm{CS}+$ and $\mathrm{CS}-$ during discriminative avoidance learning. These thalamic nuclei receive major afferent input, via the mamillothalamic tract, from the mamillary nuclei of the hypothalamus. This study investigated the contribution of mamillothalamic tract fibers to behavioral learning and to thalanic training-induced neuronal activity.

The behavioral and thalamic neuronal data of rabbits with bilateral transection of the mamillothalamic tract were compared to data of rabbits with control lesions (sham and attempted mamillothalamic tract lesions which missed their targets). A second comparison group contained rabbits not given lesions.

The mamillothalamic tract transection slowed the rate of behavioral acquisition and reduced the efficiency of avoidance performance in traincd rabbits. When trained, the transected rabbits exhibited an average success rate of avoidances on $61 \%$ of CS + trials, significantly less than the success rates $(84 \%$ and $83 \%)$ exhibited respectively by rabbits with control and no lesions. A similar performance loss occurred previously in trained rabbits given avoidance training after bilateral anterior thalamic lesions (Gabriel et al., 1983). Thus, either direct destruction or deafferentation eliminated the specific contribution to avoidance behavior made by anterior thalamic neurons: maintenance but not original acquisition of the behavior. Moreover, deafferentation eliminated the neuronal changes (excitatory and discriminative training-induced activity) found in the AV nucleus of intact animals during behavioral acquisition. These results indicated that afferent mamillothalamic tract information received by the anterior thalamic nuclei is essential both for AV thalamic traininginduced activity and for the behavioral contribution to learning made by anterior thalamic neurons. The fact that the mamillothalamic tract transections were of such different size and location compared to the anterior thalamic lesions, yet nevertheless yielded a similar and quite specific behavioral deficit, indicates that the behavioral deficit was not due to nonspecific or remote effects of the lesions.

Lesion-related changes of the neuronal activity occurred both in transected rabbits and in rabbits with control lesions. However, certain lesion-induced changes were of significantly greater magnitude in the transected rabbits. They included loss of AV thalamic tone-elicited neuronal activation and loss of traininginduced discriminative neuronal activity. The fact that these changes were greater in transected rabbits than in rabbits with control lesions suggests that the mamillothalamic tract damage was primarily responsible for them, and that the control lesions were partially effective because they disrupted mamillothalamic tract transmission to a certain extent. However, a possible contribution of nonmamillothalamic tract fiber systems to these changes cannot be ruled out. Nonmamillothalamic tract fiber system damage may have been involved as well in the losses of AV thalamic spontaneous background neuronal activity and excitatory training-induced activity (increments of elicited activity during avoidance training compared with activity during preliminary training), which occurred equivalently in transected and control lesion rabbits, relative to the levels of these activities in the no-lesion group.

In summary, the effects that were specific to mamillothalamic tract transection (i.e., were absent or significantly less robust in rabbits with control lesions) included reduction of avoidance re- sponding, abolition of discriminative training-induced activity in limbic thalamus and reduction of the magnitude of CS-elicited AV thalamic neuronal discharges. These transection-specific effects suggested that the mamillothalamic tract is involved specifically in avoidance-relevant processing of the CS+.

Other findings suggested that the system of cholinergic fibcrs which originate in the lateral dorsal tegmental (LDT) nucleus (Hoover and Baisden, 1980; Satoh and Fibiger, 1986; Woolf and Butcher, 1986; Hallanger et al., 1987; Cornwall et al., 1990; Shibata, 1992; Poremba et al., 1994) is also involved in the mediation of training-induced activity. However, this system appears to be involved only in the production of excitatory training-induced activity in the AV nucleus, as suggested by the finding that blockade of muscarinic receptors by systemic and local intracranial administration of scopolamine hydrobromide eliminated the excitatory CS-elicited activity of AV thalamic and posterior cingulate cortical neurons in trained rabbits (Henzi et al., 1990; Kubota et al., 1993) but did not affect discriminative training-induced activity.

These results considered along with the present findings suggested that excitatory training-induced activity in limbic thalamus may require jointly, inputs from both the mamillothalamic tract and the cholinergic LDT fiber systems. The cellular mechanism for excitatory training-induced activity could be analogous to "activity-dependent facilitation" (see Carew et al., 1984; Hawkins, 1991), which might be mediated by anterior thalamic $\mathrm{M}_{2} \mathrm{ACh}$ receptors that have been shown to increase in number in correspondence with excitatory training-induced activity during discriminative avoidance conditioning (Vogt et al., 1991).

Other studies indicated that cholinergic fibers which originate in the LDT nucleus run a parallel course in close proximity to the mamillothalamic tract (Satoh and Fibiger, 1986). These results are compatible with the hypothesis that the lesions in transected and control lesion rabbits disrupted these fiber systems, accounting for the nearly equivalent losses of excitatory training-induced activity in these two groups.

The loss of discriminative training-induced activity in transected rabbits could be interpreted as an indication that the anterior thalamic discriminative training-induced activity found in intact rabbits is intrinsic to the $\mathrm{AV}$ nucleus but requires mamillothalamic tract afferent input for its development. The logical alternative is that the discriminative training-induced activity is projected to the AV nucleus via direct neurotransmission from the mamillary nuclei. The latter hypothesis is supported by recent findings indicating that discriminative training-induced activity develops in multiunit recordings made in the mamillary nuclei (Kubota et al., 1994). This training-induced activity was exclusively of the late-developing variety (i.e., it did not develop until the criterial session of acquisition), the very same variety of discriminative training-induced activity which is characteristic of the AV nuclei of intact rabbits (Gabriel et al., 1977, 1980; Foster et al., 1980).

These considerations raise the question of whether the latedeveloping discriminative training-induced activity develops de novo in the mamillary nuclei or is projected there from upstream structures and relayed onward to the anterior nuclei.

The dorsal and posterior subicular complex is a principal cerebral cortical site of origin of axonal projections to the AV thalamic and mamillary nuclei (Meibach and Siegel, 1977; Swanson and Cowan, 1977; Köhler, 1990; Witter and Groenewegen, 1990). Bilateral electrolytic subicular complex lesions enhanced $\mathrm{AV}$ thalamic discriminative training-induced activity 
(Gabriel et al., 1987) indicating that the subicular afferents are not essential for training-induced activity production but may instead provide an inhibitory modulation of the training-induced activity.

Bilateral lesions of the amygdaloid complex (Poremba and Gabriel, 1991), or of the medial geniculate nucleus (Poremba and Gabriel, 1993), an origin of fibers which project to amygdaloid and periamygdaloid regions (LeDoux et al., 1985), eliminated behavioral acquisition as well as discriminative and excitatory training-induced activity in the AV and MD thalamic nuclei. Medial geniculate and amygdaloid neurons exhibit discriminative training-induced activity (Gabriel et al., 1975; Pascoe and Kapp, 1985; Maren et al., 1991). This training-induced activity was of the early-developing variety. That is, the discriminative training-induced activity in the basolateral amygdaloid nucleus attained maximum magnitude in the session of the first significant behavioral discrimination and subsequently decreased in magnitude as criterion was reached (Maren et al., 1991). If the amygdaloid and/or the geniculate training-induced activity induced mamillary and anterior thalamic training-induced activity by direct synaptic neurotransmission, then the latter training-induced activity should also appear at maximum magnitude during the session of the first significant behavioral discrimination. However, as mentioned, the mamillary and anterior thalamic training-induced activity is of the late-developing variety and therefore not a product of direct neurotransmission from amygdaloid and/or medial geniculate neurons.

Lesion studies indicate that several nuclei of the amygdala are essential for acquisition of aversively motivated conditioned responses (Kapp et al., 1990; LeDoux, 1990; Davis, 1992; Hatfield et al., 1992; Helmstetter, 1992). Yet, amygdaloid neurons are apparently not involved in maintenance of aversively motivated behavior in well-trained subjects, as indicated by studies which have shown that learned behaviors dependent on the integrity of the amygdala nevertheless survive amygdaloid lesions induced after the behavior is acquired (reviewed by Sarter and Markowitsch, 1985; see also Miserandino et al., 1990; Roozendaal et al., 1993). We offer the suggestion that the mamillothalamic and anterior thalamic system is the system which supports the maintenance of aversively motivated responding, this based on the striking ubiquity of late-developing discriminative activity in this system and, as shown here and previously, the attenuation of discriminative behavior in the late training stages in animals with mamillothalamic tract and anterior thalamic lesions. It is further suggested that the early-developing discriminative training-induced activity found in the amygdala may act as a precipitating event or trigger which initiates the late-developing mamillary and anterior thalamic training-induced activity. Perhaps this triggering function requires as a precondition, the projection of discriminative training-induced activity from the amygdala to the mamillary and anterior nuclei during the early training stages. The afferents which originate in the amygdala and which initiate the late-developing discriminative training-induced activity in the mamillary nuclei could gain access to the mamillary nuclei via amygdalofugal fibers which project to various hypothalamic areas including lateral hypothalamic area, premamillary nucleus, and supramamillary nucleus (Hopkins and Holstege, 1978; Krettek and Price, 1978; Price and Amaral, 1981; Caffé et al., 1987; Canteras et al., 1992) and thence forward to the mamillary nuclei (Gonzalo-Ruiz et al., 1992).

\section{References}

Bramham CR (1992) Opioid receptor dependent long-term potentiation: peptidergic regulation of synaptic plasticity in the hippocampus. Neurochem Int 20:441-455.

Brogden WJ, Culler FA (1936) A device for motor conditioning of small animals. Science 83:269.

Buchwald J, Holstein SB, Weber DS (1973) Multiple unit recording: technique, interpretation, and experimental applications. In: Bioelectric recording techniques, $\mathrm{Pt} \mathrm{A}$, Cellular processes and brain potentials (Thompson RF, Petterson MM, eds), pp 202-242. New York: Academic.

Caffé AR, van Leeuwen FW, Luiten PGM (1987) Vasopressin cells in the medial amygdala of the rat project to the lateral septum and ventral hippocampus. J Comp Neurol 261:237-252.

Canteras NS, Simcrly RB, Swanson LW (1992) Connections of the posterior nucleus of the amygdala. J Comp Neurol 324:143-179.

Carew TJ, Hawkins RD, Abrams TW, Kandel ER (1984) A test of Hebb's postulate at identified synapses which mediate classical conditioning in Aplysia. J Neurosci 4:1217-1224.

Colley PA, Routtenberg A (1993) Long-term potentiation as synaptic dialogue. Brain Res Rev 18:115-122.

Cornwall J, Cooper JD, Phillipson OT (1990) Afferent and efferent connections of the laterodorsal tegmental nucleus in the rat. Brain Res Bull 25:271-284

Davis M (1992) The role of the amygdala in fear and anxiety. Annu Rev Neurosci 15:353-375.

Donovick PJ (1974) A metachromatic stain for neural tissue. Stain Technol 49:49-51.

Foster K, Orona E, Lambert RW, Gabriel M (1980) Early and late acquisition of discriminative neuronal activity during differential conditioning in rabbits: specificity within the laminae of cingulate cortex and the anteroventral thalamus. J Comp Physiol Psychol 94:1069 1086.

Fox CA, Eichman J (1959) A rapid method for locating intracerebral electrode tracks. Stain Technol 34:39-42.

Gabriel M (1993) Discriminative avoidance learning: a model system. In: Neurobiology of cingulate cortex and limbic thalamus: a comprehensive handbook (Vogt BA, Gabriel M, eds). Boston: Birkhauser.

Gabriel M, Moore J (1990) Learning and computational neuroscience: foundations of adaptive networks. Cambridge, MA: Bradford Division of the MIT Press.

Gabriel M, Saltwick SE, Miller JD (1975) Conditioning and reversal of short-latency multiple unit responses in the rabbit medial geniculate nucleus. Science 189:1108-1109.

Gabriel M, Miller JD, Saltwick SE (1977) Unit activity in cingulate cortex and anteroventral thalamus of the rabbit during differential conditioning and reversal. J Comp Physiol Psychol 91:423-433.

Gabriel M, Foster K, Orona F. (1980) Interaction of laminae of the cingulate cortex with the anteroventral thalamus during behavioral learning. Science 208:1050-1052.

Gabriel M, Lambert RW, Foster K, Orona E, Sparenborg S, Maiorca RR (1983) Anterior thalamic lesions and neuronal activity in the cingulate and retrosplenial cortices during discriminative avoidance behavior in rabbits. Behav Neurosci 97:675-696.

Gabriel M, Sparenborg SP, Stolar N (1986) An executive function of the hippocampus: pathway selection for thalamic neuronal significance code. In: The hippocampus, Vol 4 (Isaacson RL, Pribram K, eds), pp 1-39. New York: Plenum.

Gabriel M, Sparenborg SP, Stolar N (1987) Hippocampal control of cingulate cortical and anterior thalamic information processing during learning in rabbits. Exp Brain Res 67:131-152.

Gabriel M, Sparenborg SP, Kubota Y (1989) Anterior and medial thalamic lesions, discriminative avoidance learning and cingulate cortical neuronal activity in rabbits. Exp Brain Res 76:441-457.

Gabriel M, Kubota Y, Sparenborg S, Straube K, Vogt BA (1991) Effects of cingulate cortical lesions on avoidance learning and traininginduced unit activity in rabbits. Exp Brain Res 86:585-600.

Girgis M, Shih-Chang W (1981) A new stereotaxic atlas of the rabbit brain. St. Louis, MO: Green.

Gonzalo-Ruiz A, Alonso A, Sanz JM, Llinás RR (1992) Afferent projections to the mamillary complex of the rat, with special reference to those from surrounding hypothalamic regions. J Comp Neurol 321: 277-299.

Hallanger AE, Levey AI, Lee HJ, Rye DB, Wainer BH (1987) The 
origins of Cholinergic and other subcortical afferents to the thalamus in the ral. J Comp Neurol 262:105-124.

Hatfield T, Graham PW, Gallagher M (1992) Taste-potentiated odor aversion learning: role of the amygdaloid basolateral complex and central nucleus. Behav Neurosci 106:286-293.

Hawkins RD (1991) Cell biological studies of conditioning in Aplysia. In: Neurobiology of learning and emotion (Madden J, ed), pp 3-28. New York: Raven.

Hawkins RD, Kandel ER, Siegelbaum SA (1993) Learning to modulate transmitter release: themes and variations in synaptic plasticity. Annu Rev Neurosci 16:625-665.

Helmstetter FJ (1992) Contribution of the amygdala to learning and performance of conditional fear. Physiol Behav 51:1271-1276.

Henzi V, Kubota Y, Gabriel M (1990) Scopolamine but not haloperidol disrupts training-induced neuronal activity in cingulate cortex and limbic thalamus during learning in rabbits. Brain Res 518:107-114.

Hoover DB, Baisden RH (1980) Localization of putative cholinergic neurons innervating the anteroventral thalamus. Brain Res Bull 5:519-524.

Hopkins DA, Holstege G (1978) Amygdaloid projections to the mesencephalon, pons and medulla oblongata in the cat. Exp Brain Res 32:529-547.

Huynh H, Feldt LS (1976) Estimation of the Box correction for degrees of freedom from sample data in randomized block and split-plot designs. J Ed Stat 1:69-82.

Johnston D, Williams S, Jaffe D, Gray R (1992) NMDA-receptor-independent long-term potentiation. Annu Rev Physiol 54:489-505.

Kapp BS, Wilson A, Pascoe JP, Supple W, Whalen PJ (1990) A neuroanatomical systems analysis of conditioned bradycardia in the rabbit. In: Learning and computational neuroscience: foundations of adaptive networks (Gabriel M, Moore J, eds), pp 53-90. Cambridge, MA: Bradford Division of the MIT Press.

Köhler C (1990) Subicular projections to the hypothalamus and brainstem: some novel aspects revealed in the rat by the anterograde $\mathrm{Pha}$ seolus vulgaris leukoagglutinin (PHA-L) tracing method. In: Progress in brain research, Vol 83 (Storm-Mathisen J, Zimmer J, Ottersen OP, eds), pp 59-69. New York: Elsevier.

Krettek JE. Price JL (1978) Amygdaloid projections to subcortical structures within the basal forebrain and brainstem in the rat and cat J Comp Neurol 178:225-254.

Kubota Y, Mayo J, Gabriel M (1993) Brainstem cholinergic projections and limbic thalamic training-related neuronal activity during avoidance learning in rabbits. Soc Neurosci Abstr 19:802.

Kubota Y, Burkybile C, Gabriel M (1994) Training-related neuronal activity in the mamillary complex and brainstem cholinergic nuclei during avoidance learning in rabbits. Soc Neurosci Abstr 20:797.

LeDoux JE (1990) Information flow from sensation to emotion: plasticity in the neural computation of stimulus value. In: Learning and computational neuroscience: foundations of adaptive networks ( $\mathrm{Ga}-$ briel M, Moore J, eds), pp 3-51. Cambridge, MA: Bradford Division of the MIT Press.

LeDoux JE, Ruggiero DA, Reis DJ (1985) Projections to the subcortical forebrain from anatomically defined regions of the medial geniculate body in the rat. J Comp Neurol 242:182-213.

Madison DV, Malenka RC, Nicoll RA (1991) Mechanisms underlying long-term potentiation of synaptic transmission. Annu Rev Neurosci 14:379-397.

Maren S, Poremba A, Gabriel M (1991) Basolateral amygdaloid multiunit neuronal correlates of discriminative avoidance learning in rabbits. Brain Res 549:311-316.
Massicotte G, Baudry M (1991) Triggers and substrates of hippocampal synaptic plasticity. Neurosci Biobehav Rev 15:415-423.

Meibach RC, Siegel A (1977) Thalamic projections of the hippocampal formation: evidence for an alternate pathway involving the internal capsule. Brain Res 134:1-12.

Miserandino MJD, Sananes CB, Mella KR, Davis M (1990) Blocking of acquisition but not expression of conditioned fear-potentiated startle by NMDA antagonists in the amygdala. Nature 345:716-718.

Pascoe JP, Kapp BS (1985) Electrophysiological characteristics of amygdaloid central nucleus neurons during Pavlovian fear conditioning in the rabbit. Behav Brain Res 16:117-133.

Poremba A, Gabriel M (1991) Amygdala lesions block acquisition of discriminative active avoidance learning in rabbits. Soc Neurosci Abstr 17:325

Poremba A, Gabriel M (1993) Lesions of the medial geniculate nucleus impair avoidance learning, limbic thalamic and cingulate cortical training-induced neuronal activity in rabbits. Soc Neurosci Abstr 19: 802.

Poremba A, Kubota Y, Gabriel M (1994) Afferent connections of the anterior thalamus in rabbits. Brain Res Bull 33:361-365.

Price JL, Amaral DG (1981) An autoradiographic study of the projections of the central nucleus of the monkey amygdala. J Neurosci 1:1242-1259.

Rescorla RA (1967) Pavlovian conditioning and its proper control procedures. Psychol Rev 74:71-80.

Roozendaal B, Koolhaas JM, Bohus B (1993) The central amygdala is involved in conditioning but not in retention of active and passive shock avoidance behavior in male rats. Behav Neural Biol 59:143149.

Sarter M, Markowitsch IIJ (1985) Involvement of the amygdala in learning and memory: a critical review, with emphasis on anatomical relations. Behav Neurosci 99:342-380.

Satoh K, Fibiger HC (1986) Cholinergic neurons of the laterodorsal tegmental nucleus: efferent and afferent connections. J Comp Neurol 253:277-302.

Shen CL (1983) Efferent projections from the mammillary complex of the guinea pig: an autoradiographic study. Brain Res Bull 11:43-59.

Shibata H (1992) Topographical organization of subcortical projections to the anterior thalamic nuclei in the rat. $\mathbf{J}$ Comp Neurol 323:117127.

Sparenborg S, Gabriel M (1992) Local norepinephrine depletion and learning-related neuronal activity in cingulate cortex and anterior thalamus of rabbits. Exp Brain Res 92:267-285.

Swanson LW, Cowan WM (1977) An autoradiographic study of the organization of the efferent connections of the hippocampal formation in the rat. J Comp Neurol 172:49-84.

Vogt BA, Gabriel M, Vogt LJ, Poremba A, Jensen EL, Kubota Y, Kang $\mathrm{E}$ (1991) Muscarinic receptor binding increases in anterior thalamus and cingulate cortex during discriminative avoidance learning. J Neurosci $11: 1508-1514$.

Winer BJ (1962) Statistical principles in experimental design. New York: McGraw-Hill.

Witter MP, Groenewegen HJ (1990) The subiculum: cytoarchitectonically a simple structure, but hodologically complex. In: Progress in brain research, Vol 83 (Storm-Mathisen J, Zimmer J, Ottersen OP, eds), pp 47-58. New York: Elsevier.

Woolf NJ, Butcher LL (1986) Cholinergic systems in the rat brain. III. Projections from the pontomesenecphalic tegmentum to the thalamus, tectum, basal ganglia, and basal forebrain. Brain Res Bull 16:603637. 